Revue d'histoire de l'Amérique française

\title{
Sous-entendus, contradictions et silences narratifs : une relecture du récit attribué à Marquette dans Thévenot (1681)
}

\section{Catherine Broué}

Volume 72, numéro 4, printemps 2019

URI : https://id.erudit.org/iderudit/1065045ar

DOI : https://doi.org/10.7202/1065045ar

Aller au sommaire du numéro

\section{Éditeur(s)}

Institut d'histoire de l'Amérique française

\section{ISSN}

0035-2357 (imprimé)

1492-1383 (numérique)

Découvrir la revue

\section{Citer cet article}

Broué, C. (2019). Sous-entendus, contradictions et silences narratifs : une relecture du récit attribué à Marquette dans Thévenot (1681). Revue d'histoire de l'Amérique française, 72(4), 61-86. https://doi.org/10.7202/1065045ar

\section{Résumé de l'article}

Le caractère lacunaire des sources historiques sur l'exploration jésuite du Mississippi entre 1672 et 1674 a conduit bien des chercheurs à s'interroger sur la paternité et la fiabilité de ces textes, dont une version a été publiée en 1681 par un éditeur de récits de voyages, Melchisédech Thévenot, à qui certains chercheurs ont imputé les incohérences qui s'y trouvent. Un examen attentif de la dimension implicite de ce récit met en lumière sa construction délibérément ambigüe. Cet examen permet d'esquisser un scénario de voyage complexe où figurent au moins deux missionnaires et impliquant davantage d'étapes (voire, d'expéditions) que ce que l'on a cru jusqu'ici, notamment une incursion sur le Missouri. Le retour du récollet Louis Hennepin de son périple à l'ouest des Grands Lacs et la découverte imminente de l'embouchure de ce fleuve par la troupe de Cavelier de La Salle pourraient expliquer que la Compagnie de Jésus ait voulu s'assurer la primeur d'au moins une partie d'une exploration entamée par Claude Allouez, mais passée sous silence jusqu'alors. 


\title{
Sous-entendus, contradictions et silences narratifs : une relecture du récit attribué à Marquette dans Thévenot (I68I)
}

\author{
Catherine Broué \\ Université du Québec à Rimouski
}

\begin{abstract}
RÉsumÉ - Le caractère lacunaire des sources historiques sur l'exploration jésuite du Mississippi entre 1672 et 1674 a conduit bien des chercheurs à s'interroger sur la paternité et la fiabilité de ces textes, dont une version a été publiée en 1681 par un éditeur de récits de voyages, Melchisédech Thévenot, à qui certains chercheurs ont imputé les incohérences qui s'y trouvent. Un examen attentif de la dimension implicite de ce récit met en lumière sa construction délibérément ambigüe. Cet examen permet d'esquisser un scénario de voyage complexe où figurent au moins deux missionnaires et impliquant davantage d'étapes (voire, d'expéditions) que ce que l'on a cru jusqu'ici, notamment une incursion sur le Missouri. Le retour du récollet Louis Hennepin de son périple à l'ouest des Grands Lacs et la découverte imminente de l'embouchure de ce fleuve par la troupe de Cavelier de La Salle pourraient expliquer que la Compagnie de Jésus ait voulu s'assurer la primeur d'au moins une partie d'une exploration entamée par Claude Allouez, mais passée sous silence jusqu'alors.
\end{abstract}

ABSTRACT - The incomplete nature of the historic narrative sources related to the jesuit exploration of the Mississippi river basin between 1672 and 1674 has led several scholars to reflect on the authorship and reliability of these texts, a version of which was published in 1681 by an editor specializing in accounts of travel and exploration, Melchisédech Thévenot, to whom some of these scholars have attributed the inconsistencies of this version. A detailed analysis of the implicit dimension of this account, highlights its deliberately ambiguous construction and phrasing. The analysis allows us to sketch a more complex travel scenario featuring at least two jesuit missionaries and more stages (possibly expeditions) than what scholars have inferred up to now, including an incursion on the Missouri river. The safe return of the récollet Louis Hennepin from his journey to the Mississippi river and the imminent discovery of the mouth of this river by Cavelier de La Salle's group could explain 
the fact that the Society of Jesus wanted to ensure that it would be credited for at least a part of the explorations accomplished by Claude Allouez, about which the public had not heard until this time.

But history, or rather historians, may err, as appears in the present case. In fact, if the gathering and furnishing of information has any weight in deciding the question of leadership, then the man to be considered before Marquette is Allouez. From this missionary the government received far more information concerning the great river; he was just as much interested in having it explored; and there is great probability that he had stood on the banks of the river before Jolliet and Marquette reached it.

F. B. Steck, The Jolliet-Marquette Expedition, 1673, p. 255.

$\mathbf{E}^{n}$ 1681 paraissait, dans le troisième Recueil de Voyages compilé par Melchisédech Thévenot ${ }^{1}$, un récit intitulé Decouverte de quelques pays et nations de l'Amerique septentrionale rapportant l'exploration du Mississippi entreprise moins de dix ans auparavant par Louis Jolliet et un narrateur écrivant au «je», non explicitement nommé dans le texte même, mais associé, dans la table des matières et l'avis au lecteur de ce même Recueil, au jésuite Jacques Marquette. Bien que ce récit ait été effectivement considéré, jusqu'au début du $\mathrm{XX}^{\mathrm{e}}$ siècle, comme le «journal» du père Jacques Marquette ${ }^{3}$, cette attribution a été mise en doute en 1928 par Francis B. Steck ${ }^{4}$ qui, relevant les nombreuses incohérences qui surgissent quand on compare l'ensemble des sources sur cette expédition les unes aux autres, faisait l'hypothèse que c'était Jolliet, et non Marquette, qui en

1. Melchisédech Thévenot, Recueil de voyage de M. Thévenot (Paris, Étienne Michallet, 1681). L'exemplaire consulté (en ligne) est celui de la bibliothèque de Gand (Belgique).

2. Ce titre figure au début du récit lui-même, Recueil, p. 1. Néanmoins, deux autres titres lui font concurrence dans le même volume: Decouverte dans l'Amerique Septentrionale par le P. Marquette jesuite, dans la table des matières au début du volume (non paginée); Voyage et découverte du P. Marquette \& du $S^{r}$ Jolliet dans l'Amerique Septentrionale, dans l'index général des trois premiers volumes, p. 16.

3. La restitution aux jésuites, en 1847 , d'une version manuscrite plus longue de ce récit avait toutefois déjà quelque peu désacralisé la version imprimée en 1681, dès lors considérée comme moins complète. Voir Lucien Campeau, "Regard critique sur la Narration du P. Jacques Marquette», Les Cahiers des Dix, 46 (1991), p. 22.

4. Francis Borgia Steck, The Jolliet-Marquette Expedition of 1673 (Glendale, California, The Arthur H. Clark Company, 1928). 
avait inspiré le fond, tandis que Dablon en était l'auteur's. Rapidement décriée par d'autres éminents historiens ${ }^{6}$, cette hypothèse n'a pas été retenue, non plus que les observations qui l'avaient motivée. Pourtant, certaines de ces observations étaient non seulement pertinentes, mais cruciales $^{7}$, en ce qu'elles montraient la complexité des liens qu'entretiennent les unes avec les autres les sources historiques sur l'expédition de 1673. Surtout, elles remettaient en question non seulement la paternité du texte publié par Thévenot, mais aussi la fiabilité de l'ensemble des sources sur l'expédition de 1673. Il me semble important de revenir sur cette question, qui est loin d'être close.

La première étape de cette ambitieuse entreprise sera, dans le présent article, de se détacher du sens littéral du texte publié dans Thévenot pour mieux comprendre les sous-entendus ou les silences qu'il installe. Il s'agira donc de relire le récit de 1681 en tenant compte de ses ambigüités et incohérences au lieu d'en faire abstraction ou d'essayer de les résoudre, afin de distinguer ce qui y est dit de ce qui y est suggéré, d'en convoquer les dimensions implicites pour mieux en éclairer les zones d'ombre. De fait, de nombreux extraits de ce texte, considéré ici comme une étape de réécriture en regard des versions manuscrites plus longues connues aujourd'hui, contiennent une forte charge implicite. Leur analyse révèle une écriture intrinsèquement ambigüe misant sur la capacité d'inférence des lecteurs extérieurs à la Compagnie de Jésus et permettant une double lecture selon que ces derniers auraient lu ou non les Relations des jésuites publiées antérieurement (ou qu'ils s'en souviendraient).

Pour mettre au jour les inférences auxquelles peuvent mener ces passages du récit attribué à Marquette en $1681^{8}$ et éclairer les contradictions

5. Francis Borgia Steck, Marquette Legends (New York, Pageant Press Inc., 1960), p. 87. Malheureusement, Steck appuie ses conclusions sur un raisonnement qui ne prend pas en compte toutes les éventualités possibles pouvant expliquer les incongruités qu'il remarque; de plus, certains de ses arguments découlent de la certitude que la lettre dite de Jolliet du 10 octobre 1674 est bien de Jolliet ; or l'authenticité de cette lettre n'est pas plus avérée que celle des autres documents (nous ne disposons que d'une seule copie non autographe). Voir Jean Delanglez, Louis Jolliet. Vie et voyages (1645-1700) (Montréal, Éditions Granger, 1950), p. 90.

6. Cette question a particulièrement interpellé les historiens jésuites Gilbert J. Garraghan, Jean Delanglez, Raphaël N. Hamilton et Lucien Campeau. Ce dernier, dans son article "Regard critique», affirme que «[1]'obscurité provient surtout d'interventions maladroites de quelques historiens» (p. 21), historiens dans lesquels il englobe surtout Steck et Delanglez. Pour une courte synthèse historiographique des débats suscités par la thèse de Steck, voir Raphaël N. Hamilton, Marquette's Explorations. The Narratives Reexamined (Madison, The University of Wisconsin Press, 1970), p. 43-45.

7. Je reviens dans le présent article sur quelques-unes de ces observations.

8. Les mêmes passages se retrouvent dans les copies manuscrites de la Relation pour les années 1673 à 1678 conservées dans les archives jésuites à Vanves (Paris) et à Montréal (Canada), mais l'étude comparative de ces sources devra faire l’objet d'un travail ultérieur. 
qu'ils présentent en regard d'autres passages du même récit, il s'agira d'abord de situer ce texte par rapport au contexte de publication, d'une part, et par rapport aux autres versions jésuites - toutes manuscrites - de l'expédition, d'autre part. Un retour sur les informations données à lire dans les Relations des jésuites jusqu'en 1672 sur l'ouest des Grands Lacs, qui établissaient les objectifs de l'exploration à venir, nous permettra ensuite de mieux comprendre le contexte d'énonciation sans lequel des dimensions implicites du récit de 1681 ne peuvent être saisies. La construction même du texte et les procédés littéraires qu'ils convoquent seront enfin examinés : l'hérö̈sation des protagonistes, l'imprécision des données géographiques et temporelles, l'abondance des descriptions, la présence/ absence du missionnaire jésuite Claude Allouez, jointes à quelques incongruités de la narration, détournent l'attention du lecteur et l'empêchent de trop s'attarder sur l'indigence narrative de ce récit.

\section{DES MISSIONS MENACÉES}

La publication, en 1681, du récit de l'exploration au Mississippi de 1673 survient à un moment crucial pour les missions de la Compagnie de Jésus, en particulier celles de l'ouest des Grands Lacs. Les entreprises d'exploration de René-Robert Cavelier de La Salle, entamées depuis 1678, avaient compromis plusieurs de ces missions implantées depuis la «baie des Puants» (Green Bay) chez des peuples Illinois, Miamis et «d'autres nations du Sud»". Très hostile à l'égard de la Compagnie de Jésus et en particulier envers Claude Allouez, principal pionnier des missions de l'Ouest, La Salle avait tenté, à plusieurs reprises, de dénigrer leur travail missionnaire ${ }^{10}$ auprès de ses correspondants et du gouverneur Frontenac, lui-même notoirement critique des jésuites.

Qui plus est, les entreprises de La Salle suivaient exactement le programme de colonisation esquissé en 1674 par le supérieur jésuite Claude

9. Dans sa relation, Henri Joutel signale que l'arrivée aux Illinois de leur petit groupe en provenance de la colonie installée par La Salle sur les rives du Texas a suscité la frayeur du jésuite Claude Allouez, Archives nationales de France (AN), MAR 3JJ 276, Relation du voyage de Mr de la Salle dans le nord de l'Amerique Septen ${ }^{a l e}$ en 1684 pour y faire un etablissement dans la partie qu'il en avoit auparavant découverte.

10. Voir la correspondance de La Salle conservée à la Bibliothèque nationale de France (BNF), Fonds français, Clairambault 1016, mais aussi le récit rédigé par l’ingénieur Jean-Baptiste Minet en 1684-1685, à partir du témoignage de Gabriel Barbier et Nicolas de La Salle, qui avaient participé aux entreprises de 1678-1681 et de 1681 à 1683, Bibliothèque et Archives du Canada (BAC), MG18-B19, Voiage fait par dedans les terres du Sud dans l'année 1682. 
Dablon dans sa Relation de la $\mathrm{Mer} d u S u d^{11}$ rédigée à partir du compte rendu oral de Louis Jolliet:

$\mathrm{La} 4^{\mathrm{e}}$ remarque regarde un aduantage grande $\&$ considerable $\&$ qu'on aura peut estre de la paine a croire: c'est que nous pourrions aissem[en]t aller iusques a la floride en barque $\&$ par une fort belle navigation il ny auroit qu'une saignée a faire coupant demilieue de prairie seulem[en]t pour entrer du fond du lac des Ilinois dans la riuiere St Louis, voicy la route qu'on tiendroit la barque se deuroit faire dans le lac Erié qui est proche de Lontario, elle iroit aisement du lac Erié dans le lac huron d'où elle entreroit dans le lac Ilinois au fond duquel on feroit la tranchée ou le Canal dont i’ay parlé pour auoir passage dans la riuiere de St Louis qui se descharge dans Messissipi y estant la barque navigable facillemt iusqu'au Golphe de Mexique, Le fort de Cararokoui que Monsr le Comte de fontenac a fait construire sur Lontario fauoriseroit beaucoup cette entreprisse parcequ'il faciliteroit la communication de Quebec au lac Erié d'où ce fort n'est pas beaucoup esloigné $\&$ sans une chutte d'eau qui separe le lac Erié d'auec celuy de L'ontario la barque qui se bastiroit a Catorokoui pourroit aller jusques a la floride par les routtes que ie vient de marquer. La $5^{\mathrm{me}}$ remarque est touchant les grands avantages qu'il y a d'establir de nouvelles Collonies dans de si beaux pays, $\&$ sur des terres si fertiles ${ }^{12}$.

La construction d'un premier navire en amont des chutes du Niagara, en 1678 , et d'un second sur la rivière Illinois, en $1679^{13}$, montre que Frontenac avait retenu le programme de colonisation suggéré par Dablon; en cédant le fort Frontenac à Cavelier de La Salle et en choisissant ce dernier pour mettre en œuvre ces suggestions ${ }^{14}$, il avait toutefois délibérément mis à l'écart la Compagnie de Jésus et, du même coup, Louis Jolliet. Cette prise en main de l'exploration de l'ouest des Grands Lacs par le gouverneur de la Nouvelle-France correspondait à une volonté politique de Louis XIV, soucieux de minimiser l'influence de cet ordre dans la colonie depuis 1663 , mais ne s'était pas encore vraiment fait sentir sous

11. Pour un relevé des cinq copies de la Relation de la descouverte de plusieurs pays scitués au midy de la Nouvelle France faitte $A^{\circ} 1673$ (le titre est variable selon les copies), voir J. Delanglez, Louis Jolliet, p. 89-96.

12. AN, MAR 3JJ-68, Relation de la descouverte de plusieurs pays scitués au midy de la Nouvelle France faitte $A^{\circ} 1673$, dossier 16, $f^{\circ} 8-10$. Cette version compte parmi les cinq copies que nous connaissons de la relation envoyée par Claude Dablon à Paris le $1^{\text {er }}$ août 1674 . Elle est convoquée ici parce qu'elle est plus complète que les autres versions pour ce passage.

13. Louis Hennepin, Description de la Louisiane, nouvellement découverte au sud de la Nouvelle-France (Paris, veuve Sébastien Huré, 1683).

14. La permission royale de «descouvrir la partie occidentalle de la Nouvelle France» est accordée à Cavelier de La Salle en 1678. Elle comprenait l'autorisation de construire autant de forts qu'il le jugerait bon sur son passage, Archives nationales d'Outre-Mer (ANOM), Colonies, F3-5, fo 40-41. 
l'administration coloniale précédente ${ }^{15}$. Enfin, la construction de quelques forts et de plusieurs entrepôts par la troupe de La Salle et l'exacerbation des conflits entre Iroquois, Miamis et Illinois survenue sur son passage ${ }^{16}$ avaient fragilisé les missions de l'Ouest. Une note de la main de Claude Dablon, supérieur des missions de la Nouvelle-France jusqu'en 1678, à la fin du Recit des Voyages et des decouvertes du pere Jacques Marquette de la Compagnie de Jesus en l'année 1673 et aux suivantes, laisse transparaître l'inquiétude de Dablon pour l'avenir de ces missions:

L'année d'après à scavoir en 1678 Le P. Allouez partit pour retourner en cette mission, et pour y demeurer 2 ans de suitte, affin d'y travailler plus solidement a la conversion de ces peuples. Nous avons appris du depuis que les Iroquois ont fait une incursion jusques là, mais qu'ils ont esté battus par les Ilinois: c'est ce qui va bien eschauffer la guerre entre ces Nations, et est pour beaucoup nuire a cette Mission, si Dieu n'y met la main ${ }^{17}$.

En outre, les récollets, dont le zèle missionnaire n'avait rien à envier à celui de leurs confrères jésuites et qui étaient favorisés par le gouverneur, secondaient les entreprises de La Salle depuis 1678 et étaient rapidement devenus des acteurs incontournables de l'exploration territoriale ${ }^{18}$. L'arrivée inattendue du récollet Louis Hennepin à Michillimakinak, à la fin de l'automne 1680, après un périlleux voyage jusqu'au Mississippi et peut-être au-delà, a pu d'ailleurs être perçue comme annonciatrice d'un déclin imminent des missions jésuites de l'Ouest ${ }^{19}$.

Or, la Compagnie de Jésus n'avait jamais publié les résultats de l'exploration de 1673 . Si le naufrage allégué de Jolliet ${ }^{20}$ peut expliquer que le récit de l'exploration ne soit pas parvenu immédiatement au supérieur des missions de la Nouvelle-France ${ }^{21}$, la Relation couvrant les années 1673 à

15. Gilles Havard, Empire et Métissages: Indiens et Français dans le Pays d'en Haut, 1660-1715, 2 édition (Québec, Septentrion, 2017), p. 200-220.

16. Sur cette question, voir Catherine Broué, «Errance missionnaire, errances documentaires: une Relation inédite du père Louis Hennepin?», dans Lucie Desjardins, Marie-Christine Pioffet, Roxanne Roy, Actes du Congrès de la NASSCFL 2015, Errance au XVIII siècle (Tübingen, Narr Éditeur, 2017), p. 97-116.

17. La version citée ici est celle des Archives des jésuites au Canada (AJC), cote Q-0001, 296, f 60.

18. Trois récollets l'accompagnèrent lors de sa première expédition (1678-1680), dont le père Zénobe Membré qui se vit chargé de la «mission des Illinois », sur la rivière du même nom. Or, à partir de 1671, plusieurs groupes Illinois s'étaient installés sur la rivière Illinois à la sollicitation des missionnaires jésuites.

19. De fait, les missions jésuites atteignirent leur apogée en 1679 pour décliner régulièrement jusqu'en 1702, comme le fait remarquer Louise Kellogg, The French Regime in Wisconsin and the Northwest (Madison, State Historical Society of Wisconsin, 1925), p. 169.

20. Ce naufrage est rapporté notamment dans une lettre attribuée à Jolliet datée du 10 octobre 1674 et conservée aux Archives de Saint-Sulpice à Paris, cote 19-3.

21. En 1675, Dablon semble avoir en main ce qu'il lui faut, voir L. Campeau, «Regard critique... », p. 29. 
$1678^{22}$ avait pourtant bel et bien été préparée pour publication. Augmentée de nombreux commentaires, cette Relation présentait une version très proche, sur le plan narratif, du récit proposé dans Thévenot ${ }^{23}$. Pourquoi insérer un récit d'une telle importance stratégique dans un travail de compilation réalisé par un éditeur extérieur à la Compagnie de Jésus? Le fait est qu'en publiant ce récit dans un recueil de voyage non directement lié aux Relations des jésuites, il était facile de désavouer ce texte s'il suscitait, pour une raison ou pour une autre, quelques remous. Par ailleurs, la narration à la première personne du singulier dispensait de nommer le ou les missionnaires engagés dans l'expédition; l'ajout du nom de Jacques Marquette, alors décédé, dans la table des matières, ne pouvait guère lui nuire, et l'on pourrait toujours alléguer, en cas d'objections, que l'éditeur Thévenot avait pris des libertés malencontreuses. Effectivement, d'ailleurs, on a plus d'une fois expliqué les incohérences du texte par une négligence éditoriale de Thévenot ${ }^{24}$, alors même que ce texte ne présente pas plus d’incohérences que les versions officiellement jésuites de ce récit.

Quoi qu'il en soit, l'absence de récit officiel risquant de priver définitivement la Compagnie de Jésus de toute prérogative ultérieure sur les territoires découverts, la parution du récit attribué à Marquette dans Thévenot en 1681 pouvait paraître comme un pis-aller assurant au moins à cette expédition une place dans l'histoire. Ce faisant, elle marquait toutefois une rupture avec les Relations publiées jusqu'en 1672 et avec les objectifs d'évangélisation que celles-ci laissaient entrevoir.

22. Plusieurs exemplaires existent de cette relation dans les archives jésuites. Citons ici «Pour Relation de l’année 1678 - Récit des voyages et des découvertes du P. Jacques Marquette de la Cie de Jésus en l'année 1673 et années suivantes", au R. P. Pierre de Verthamont Provincial de la Province de France, Archives françaises de la Compagnie de Jésus (AFCJ), fonds Brotier 159.1, fo 1-11.

23. Cette affirmation nécessiterait une démonstration en bonne et due forme qui fera l'objet d'un prochain article. Ce récit est-il antérieur ou postérieur à la "Relation de l'année 1678 », qui comprend un "Récit des Voyages et des Découvertes du P. Jacques Marquette de la Cie de Jésus en l'année 1673 », dont aucune des versions conservées dans les archives de la Compagnie de Jésus n’a été publiée au XVII siècle? Répondre à cette question nécessite également un long développement qui ne trouvera pas de place ici. Pour une présentation de ces versions, dont l'une est conservée aux Archives des jésuites au Canada (Montréal), et les autres, complètes ou fragmentaires, sont conservées aux Archives françaises de la Compagnie de Jésus (Vanves), voir J. Delanglez, Louis Jolliet, p. 167-182.

24. Dans sa revue des sources sur l'expédition de 1673, J. Delanglez (Louis Jolliet, p. 79-108) ne prend même pas la peine d'inclure le récit édité par Thévenot. R. N. Hamilton (Marquette's Explorations..., p. 24 et p. 134-135) considère pour sa part ce récit comme une version abrégée et fautive qui serait dérivée du «journal» de Marquette, dont on devrait les imperfections à l'éditeur. L. Campeau, très critique des travaux de Steck et de Delanglez, considère aussi que le récit publié par Thévenot dépend de l'une des versions de la "Relation de l'année 1678", qui comprend un «Récit des Voyages et des Découvertes du P. Jacques Marquette de la Cie de Jésus en l'année 1673» ("Regard critique...», p. 24 et 37), mais l'estime suffisamment valable pour en tenir compte dans sa comparaison des différentes versions (p. 43-45). 


\section{ÂMES EN PÉRIL ET CHEMIN DU JAPON - LES RELATIONS DES JÉSUITES JUSQU'EN 1672}

Les Relations annuelles publiées par la Compagnie de Jésus jusqu'en 1672 montrent, on le sait, un intérêt marqué pour la découverte d'une voie fluviale qui aurait permis de traverser le continent et de rejoindre le continent asiatique (où cet ordre religieux était déjà implanté depuis la fin du $\mathrm{XVI}^{\mathrm{e}}$ siècle). Les renseignements géographiques qu'apportent ces Relations en ce qui a trait à l'ouest des Grands Lacs sont schématiques ${ }^{25}$, mais importants: dès 1671 , les jésuites se préparent à évangéliser des nations «considérables» « au-delà» du Mississipp ${ }^{26}$. Les Illinois et d'autres nations «tirant au Sud», dont la croyance en un "génie» unique "maistre de tous les autres» pourrait les prédisposer au message chrétien ${ }^{27}$, attisent les espoirs missionnaires ${ }^{28}$.

A six ou sept journées plus bas que les Il[in]ois, il y a une autre grande Riviere dans laquelle sont des Nations prodigieuses, qui se servent de Canots de bois; nous ne pouvons pas rien en écrire autre chose $[s i c]$ jusques a l'année prochaine, si Dieu nous fait la grace de nous y conduire ${ }^{29}$.

Quand Jacques Marquette remplace Claude Allouez à la mission du Saint-Esprit en septembre 1669, il poursuit la collecte de renseignements entamée par son prédécesseur sur ces peuples nombreux et prometteurs, et tout est mis en œuvre pour bien préparer leur conversion; un jeune Illinois lui est même assigné, qui lui servira de «maître de langue» durant l'hiver $1670^{30}$. Un projet de mission au-delà du Mississippi a pris forme et Marquette a reçu l'ordre d'en établir les fondements ${ }^{31}$. Le "vrai pays ${ }^{32}$ » des Illinois étant situé au-delà d'une "grande rivière» se déchargeant "vers

25. Le secret entourant, à cette époque, les connaissances géographiques (qui conféraient un avantage politique, économique ou religieux majeur sur la concurrence) oblige à distinguer les connaissances acquises des connaissances révélées et pourrait expliquer le caractère schématique et lacunaire des renseignements fournis au public.

26. Reuben G. Thwaites, The Jesuit's Relations and Allied Documents Travels and Explorations of the Jesuit Missionaries in New France, 1610-1791, 73 vol. (Cleveland, 1896-1901), vol. 54, relation de 1670-1671, p. 166. Dorénavant, je ferai référence à cet ouvrage à l'aide de l'acronyme $J R$ suivi du numéro de volume.

27. JR, vol. 51, relation de 1667-1668, p. 44.

28. La grande mission dite «des Outaouais» devient en peu de temps, aux yeux des jésuites, «l'une des plus belles de la Nouvelle-France», JR, vol. 52, relation de 1668-1669, p. 98. Pour une description de l'état de ces missions en 1673, voir par exemple L. Kellogg, The French Regime.

29. Lettre de Marquette à Le Mercier, JR, vol. 54, 1669-1670, p. 190. Fait intéressant, une étude linguistique récente attribue à l’hydronyme «Missouri » l'acception de «canot de bois». Michael McCafferty, "Correction: Etymology of Missouri", American Speech, 79, 1 (printemps 2004), p. 32, en ligne <https:// muse.jhu.edu/article/54836>.

30. JR, vol. 54, 1669-1670, p. 166.

31. Lettre de Marquette au supérieur Le Mercier, JR, vol. 54, 1669-1670, p. 184.

32. JR, vol. 55, 1670-1671, p. 214. 
la Virginie», cette mission pourrait donner accès à une "autre grande rivière" peuplée de "très grandes nations ${ }^{33}$ ".

Aussi la mission de Saint-François-Xavier est-elle bientôt établie dans la «baie des Puants» (Green Bay) ${ }^{34}$. Cette mission permettra, selon la Relation de 1669-1670, de "porter la foi » à une nation «bien considérable 35 » vivant «vers» le Mississippi :

a quelques journees de la Mission de saint François Xavier, qui est la Baye des Puans, se trouve une grande Riviere large d'une lieuë $\&$ davantage, qui venant des quartiers du Nord, coule vers le Sud, \& si loin que les Sauvages qui ont navigé sur cette Riviere, allant chercher des ennemis a combattre, aprés quantité de journées de navigation, n'en ont point trouvé l'embouchure, qui ne peut estre que vers la Mer de la Floride, ou celle de Califournie. Il sera parlé cy aprés dune Nation bien considerable, qui habite vers cette Riviere, \& du voyage que nous esperons y faire cette annee, pour y porter la Foy, \& prendre en même temps connoissance de ces nouveaux Pays ${ }^{36}$.

Dans ce premier extrait de la Relation de 1669-1670, le cours nord-sud du Mississippi, appelé ici simplement "grande riviere», ne fait aucun doute dans l'esprit du relateur, même si ce dernier s'interroge sur la situation de son embouchure: ce fleuve se décharge-t-il dans la "Mer de la Floride», au sud (le golfe du Mexique) ou dans la "Mer de la Califournie» (le golfe de Californie ou l'océan Pacifique) à l'ouest? Les renseignements tirés des Autochtones seraient insuffisants pour en décider ${ }^{37}$, mais la question importe peu aux missionnaires, qui ont appris l'existence d'un affluent occidental qui répondrait davantage à leurs projets :

D’ailleurs, nous sommes aussi assurez par le rapport de quantité d'autres Sauvages, dont les depositions s'accordent tres-bien, qu'à deux cens lieuës de la Mission du saint Esprit, aux Outaoüaks, vers le Couchant, se trouve la Mer de l'oüest, en laquelle on descend par une autre grande Riviere, qu'on trouve a huict journées de ladite Mission, laquelle Riviere va \& vient bien avant dans les terres

33. JR, vol. 51, 1669-1670, p. 46.

34. La lettre d'Allouez à Le Mercier raconte son hivernement (de novembre 1669 à mai 1670) sur la rive occidentale de Green Bay et sur la rivière Fox, JR, vol. 54, 1669-1670, p. 166.

35. JR, vol. 54, 1669-1670, p. 136.

36. JR, vol. 54, 1669-1670, p. 136. Mes italiques.

37. Tout à l'honneur de la prudence scientifique jésuite, ce doute exprimé sur l'aboutissement du fleuve paraît néanmoins improbable, même compte tenu de la méconnaissance d’alors de la géographie nord-américaine et des difficultés techniques que présentait encore l'évaluation des longitudes : au regard des renseignements fournis par les Autochtones interrogés, le cours nord-sud du Mississippi sur une longueur équivalente à "quantité de journées de navigation" après l'embouchure de cet important affluent ouest (le Missouri?) rendait pratiquement impossible que l'on ait pu envisager sérieusement que ce fleuve se jette ailleurs que dans le golfe du Mexique. 
[...] on en peut tirer ces avantages, qu'il n'est pas impossible de passer de la Mer $d u$ Nord à celle du Sud, ou a celle du Couchant: Secondement, que cette Mer du Couchant ne pouvant estre que celle du Japon, on s'en pourroit faciliter le trajet, $\&$ ensuite le commerce ${ }^{38}$.

La distinction établie dans ce second extrait entre "Mer du Sud» (correspondant aujourd'hui à l'océan Pacifique) et "Mer de l'Ouest» nous rappelle que les géographes et les explorateurs de l'époque espéraient trouver un passage vers l'Asie par un golfe (la Mer de l'Ouest) ou un détroit (le détroit d'Anian) censé permettre de passer commodément d'un océan à un autre ${ }^{39}$. Le surgissement du toponyme «Mer du Japon» brouille un peu plus l'entendement, puisque le «Couchant» étant synonyme de l’ «Ouest», ces trois mers «de l'Ouest», «du Japon» et «du Sud» ne renvoient au final qu'à un seul espace maritime: l'océan Pacifique. L'ambigüité créée par le recours à trois appellations et deux points cardinaux différents pour faire référence à une même direction, l'ouest, pèsera lourd dans l'interprétation des textes relatifs à l'exploration de 1673 .

En dépit de ce brouillage, la Relation de 1669-1670 distingue clairement la "grande rivière » au cours nord-sud (le Mississippi) d'une «autre grande Riviere» (probablement le Missouri) coulant vers l'occident, située à «six ou sept journées plus bas que les Il[lin]ois", ces derniers résidant pour leur part sur les bords d'un affluent occidental du Mississippi situé plus près des missions jésuites ${ }^{40}$. La "nation considérable» si prometteuse vivant « vers » le Mississippi, difficile à identifier du fait de cette localisation géographique vague, est peut-être celle des Illinois mentionnée dans les Relations des jésuites antérieures, mais peut-être aussi celle des Miamis, que Claude Dablon, supérieur des missions de la Nouvelle-France, classe également parmi les nations illinoises ${ }^{41}$. Quoi qu'il en soit, cette nation non précisée non seulement offrirait un nouveau territoire de mission, mais constituerait également une étape à partir de laquelle les missionnaires pourraient recueillir de nouvelles informations sur les territoires encore à explorer et, notamment, sur un second cours d'eau important venant de l'occident. Les deux grands cours d'eau correspondent sans

38. JR, vol. 54, 1669-1670, p. 136-138. Mes italiques.

39. Lucie Lagarde, «Le Passage du Nord-Ouest et la Mer de l’Ouest dans la cartographie française du $18^{\mathrm{e}}$ siècle. Contribution à l'étude de l'œuvre des Delisle et Buache », Imago Mundi, 41 (1989), p. 19.

40. JR, vol. 54, 1669-1670, p. 188-190.

41. JR, vol. 55, 1670-1671, p. 200. Le fait que deux guides miamis, en 1673, aient aidé les voyageurs à trouver le portage menant à la rivière Wisconsin, portage servant de prélude à l'expédition, n'est peutêtre pas fortuit. Recueil, p. 8. 
doute à ce que Radisson appelait la "Rivière fourchue ${ }^{42}$ », qu'il disait constituée de deux branches aux cours respectifs sud et ouest ${ }^{43}-1$ e Mississippi et le Missouri.

La nomination d'un supérieur (Claude Dablon) pour les missions outaouaises ${ }^{44}$ indique assez l'importance que la Compagnie de Jésus entend donner à ces missions nouvelles ou à venir. En septembre 1670, Dablon visite, en compagnie d'Allouez, une bourgade peuplée de Maskoutens et d'Oumamis (aussi appelés dans les Relations Miamioueks ou plus simplement Miamis) en un lieu non précisé ${ }^{45}$. L'importance de la réception - la cérémonie du calumet - que les Miamis préparent à l'occasion de cette visite laisse croire qu'un accord déterminant est alors conclu ${ }^{46}$.

La visite de Claude Dablon dans les missions de l'Ouest en 1670-1671 aura ainsi permis de préciser le potentiel évangélique, commercial et colonial du territoire. Elle aura également permis aux missionnaires d'affiner leur connaissance du bassin mississippien et de préciser deux objectifs d'un même projet d'évangélisation : établir une mission chez les Illinois, dont le "vrai pays» était situé à l'ouest du Mississippi, et explorer un second affluent occidental situé plus au sud, susceptible de mener à l'océan Pacifique et de permettre l'évangélisation d'autres nations encore plus éloignées. Quant au Mississippi, son cours nord-sud ne semblait guère susciter la curiosité des missionnaires, malgré le doute qui demeurait quant à son embouchure.

Les sources jésuites subséquentes sur l'expédition de Jolliet ne reprendront pourtant aucun des deux objectifs évoqués plus haut. Au contraire,

42. Le journal du troisième voyage, en anglais, de Radisson, porte "the forked river», dans Louise Phelps Kellogg, Early Narratives of the Northwest (New York, Barnes \& Noble, 1953), p. 61-62.

43. Radisson suivait probablement en cela les conceptions hydrographiques de ses informateurs autochtones. De fait, la partition actuelle de ces deux cours d'eau est arbitraire : le bassin hydrographique du Mississippi comprend aussi celui du Missouri, et l'on pourrait aussi bien considérer que c'est le Mississippi qui se jette dans le Missouri et non l'inverse.

44. JR, vol. 52, 1668-1669, p. 198.

45. On présume généralement qu’il s'agit de la mission de Saint-Jacques déjà mentionnée, dont le centre était établi dans le cours supérieur de la rivière Fox, mais rien dans les Relations des jésuites ne permet d'être assuré que la cérémonie s'est déroulée à cet endroit. Dans la Relation de 1669-1670, la description du lieu de résidence des Oumamis laisse croire que le rédacteur a eu l'occasion d'en admirer le paysage: "Ces peuples sont establis en un tres-beau lieu, où l'on voit de belles Plaines, \& Campagnes à perte de veuë: leur Riviere conduit dans la grande Riviere, nommée Messipi, il n’y a que six jours de Navigation: C'est le long de cette Riviere où sont les autres nombreuses Nations ", JR, vol. 54, 1669-1670, p. 230-232.

46. Les Miamis auraient-ils permis aux jésuites, à cette occasion, de s'installer sur leur territoire? Allouez affirmera plutôt, dans la Relation de 1673-1674, avoir déjà commencé un travail d'évangélisation auprès d'un groupe kaskakia, $J R$, vol. 58, 1673-1674, p. 264, mais il est difficile de savoir où se trouvait cette mission et quand ce travail évangélique eut lieu (cette question fait l'objet d'un débat entre L. Kellogg et R. N. Hamilton, voir R. N. Hamilton, Marquette's Explorations, p. 187). 
elles laisseront entendre que le but de cette expédition était de descendre le Mississippi pour s'assurer de son cours nord-sud.

\section{ÉVANGÉLISATION ET EXPLORATION: \\ UN TOURNANT NARRATIF RADICAL EN 1674}

En regard des deux objectifs jésuites relatifs à l'ouest du Mississippi établis avant 1672, le récit offert au public en 1681 a peut-être surpris ou déçu les anciens lecteurs des Relations les plus assidus, car la narration ne répond pas aux mêmes objectifs. La mission des Illinois à laquelle Marquette se préparait depuis 1669 ne se matérialise que par un bref arrêt d'une journée dans l'un de leurs villages, au demeurant mal situé sur la rive du Mississippi, puis, sur le chemin du retour, par un passage chez des Kaskakias installés sur la rivière Illinois ${ }^{47}$. Quant à l'affluent occidental qui se trouvait à quelques jours au sud du «vrai pays» illinois, affluent qui constituait en $1670-1671^{48}$, rappelons-le, le second objectif de l'exploration, il acquiert un nom, "Pekitanoni ${ }^{49}$ ", mais reste à l'état d'espérance:

Pekitanoni est une riviere considerable, qui venant assez loin du costé du Nord-Oüest se décharge dans Mississipy; plusieurs Bourgades de Sauvages sont placées le long de cette riviere: j'espere par son moyen faire la découverte de la mer Vermeille ou Golfe de Californie ${ }^{50}$.

De fait, malgré l'importance constatée de ce cours d'eau et la certitude que le Mississippi descend très loin vers le sud, le récit de 1681 ne concerne que le fleuve et n'évoque qu'en passant les eaux bourbeuses du Missouri. Qui plus est, les voyageurs s'arrêtent en route avant même d'avoir vérifié dans quelle mer le Mississippi débouche, alors que seule l'embouchure de ce fleuve restait à préciser selon les Relations d'avant 1672. L'exploration d'une voie pouvant mener à la "mer du Sud», cet océan reliant le continent américain au continent asiatique qui devait se trouver à l'ouest de la Nouvelle-France et qui constituait le cœur du mandat de Jolliet, ne concerne plus, dans le récit de 1681, que la "mer du midy», au sud.

47. L'installation des Kaskakias à cet endroit aurait été convenue lors du séjour de Dablon dans les missions de l'ouest, si l'on en croit la Relation de 1673-1674, JR, vol. 58, 1667-1669, p. 264. Ce n'est que l'année suivante qu'aurait été fondée la «mission de la Conception » chez les Illinois kaskakias.

48. JR, vol. 55, 1670-1671, p. 214.

49. La Carte de la Nouvelle France où se voit le cours des Grandes Rivieres de S. Laurens et de Mississipi, dressée par Henri Abraham Chatelain vers 1719 (Amsterdam), assimile explicitement le Missouri à la rivière «Pekitanoni». La graphie Pekitanoui, plus juste, figure dans d'autres documents, dont l'ajout de 4 feuillets comblant une lacune du Récit des Voyages et des Decouvertes conservés aux Archives des jésuites au Canada (Q-0001, 296), f $24 \mathrm{v}$.

50. Recueil, p. 30. 


\section{INFÉRENCES, SOUS-ENTENDUS, INCOHÉRENCES ET CONTRADICTIONS}

\section{La mission illinoise: un arrêt imprévu?}

Malgré leurs imprécisions, les Relations d'avant 1672 laissent à penser que les missionnaires jésuites connaissaient la route à prendre pour se rendre chez les Illinois habitant "vers » le Mississippi et qu'ils y étaient attendus. De même, le récit de 1681 affirme d'emblée que les voyageurs s'étaient soigneusement renseignés avant de partir ${ }^{51}$. Pourtant, ce même récit fait silence sur la situation géographique de la bourgade ${ }^{52}$ où les voyageurs font un premier arrêt. Ce lieu est même si peu décrit qu'on a longtemps hésité sur sa localisation ${ }^{53}$, alors que la latitude donnée pour l'embouchure de la rivière Wisconsin dans le Mississippi montre que les voyageurs disposaient d'instruments de mesure et que leur mémoire n'était pas en cause.

Si cette imprécision sélective pourrait à la rigueur s'expliquer par le naufrage allégué par Jolliet et la perte subséquente de tous ses papiers, ce même naufrage n'explique pas la posture narrative du récit qui laisse entendre que la visite d'un village illinois sur la rive occidentale du Mississippi était fortuite, que Jolliet et Marquette ne savaient pas où ils allaient et que leur visite n'avait été aucunement préméditée. Une telle lecture est suggérée notamment par le choix de déterminants indéfinis et par les procédés héroïsants employés pour faire valoir la bravoure des protagonistes :

Enfin le vingt-cinq Juin nous apperçûmes sur le bord de l'eau des pistes d'hommes, \& un petit sentier assez battu qui entroit dans une belle prairie, nous nous arrestâmes; \& jugeant que c'estoit quelque chemin qui conduisoit à quelque Village de Sauvages, nous prismes resolution de l'aller reconnoistre. Nous laissons donc nos deux Canots sous la garde de nos gens, leur recommandant bien de ne se pas laisser surprendre; apres quoy Monsieur Joliet \& moy entreprismes cette découverte, assez hazardeuse pour deux hommes seuls, qui s'exposent à la discretion d'un peuple barbare \& inconnu. [...] les Sauvages sortent promp-

51. «L'on avoit eu le soin de tirer des Sauvages tout ce qui s'estoit pû tirer de lumiére de ces pays; l'on en avoit mesmes tracé une Carte sur leur recit, les rivieres y estoient marquées, le nom des Nations nous devions traverser, \& les rums de vent que nous devions suivre dans ce Voyage.» Recueil, p. 2-3.

52. Il faudrait employer le pluriel, puisque les Illinois avaient, au-delà du Mississippi, "cinq grands bourgs » d'après la Relation de 1669-1670, JR, vol. 54, 1669-1670, p. 166.

53. Les historiens ont longtemps hésité entre la rivière Des Moines et la rivière Iowa. La recherche archéologique des vingt dernières années a, semble-t-il, tranché en faveur de la Des Moines, voir Larry Grantham, "The Illini Village of the Marquette and Jolliet Voyage of 1673 », The Missouri Archeologist, 54 (déc. 1993), p. 1-20, cité par Daniel Hechenberger, «The Jesuits: History and Impact: From Their Origins Prior to the Baroque Crisis to Their Role in the Illinois Country", Journal of the Illinois State Historical Society, 100, 2 (été, 2007), p. 92, note 52. 
tement de leurs Cabanes, $\&$ nous ayant probablement reconnu pour François, sur tout voyant une robbe noire, ou du moins n'ayant aucun sujet d'apprehender, puisque nous n'étions que deux hommes $\&$ que nous les avions avertis de nostre arrivée; ils députent quatre Vieillards pour nous venir parler ${ }^{54}$.

Tout, dans ce passage, concourt à créer une atmosphère de mystère et d'inquiétude à partir de laquelle il est tentant d'inférer que les voyageurs allaient à l'aveuglette: ces derniers aperçoivent «un petit sentier» comme par hasard, sans qu'ils l'aient cherché en tout cas, et jugent qu'il peut conduire à "quelque village», le déterminant indéfini appuyant l'idée qu'ils ne savent pas où ce sentier mène ${ }^{55}$; ils prennent des précautions pour laisser canots et marchandises sous surveillance, et ces précautions renforcent l'idée que les voyageurs et leur chargement pourraient être interceptés par des nations hostiles dont on pourrait ignorer l'existence; enfin, la décision des chefs («M. Jolliet et moi») d’aller en éclaireurs est présentée comme un acte de bravoure, alors qu’une telle décision pourrait paraître imprudente et semble peu vraisemblable: pourquoi envoyer en éclaireurs les chefs de l'expédition, s'ils risquent véritablement leur vie ${ }^{56}$ ?

En réalité, la mention de la "robe noire» portée par le missionnaire, pour expliquer que les Illinois ne sont pas effrayés par l'arrivée soudaine de deux "François», laisse entendre que ce peuple connait cet habit et donc l'ordre qui la porte, mais de façon si discrète qu'elle échappe au lecteur qui n'aurait pas lu les Relations précédentes ou en aurait oublié la teneur. En effet, une autre raison expliquant l'accueil serein réservé aux voyageurs est immédiatement donnée: deux hommes seuls ayant en outre prévenu de leur arrivée ne peuvent constituer une menace. $\mathrm{Si}$, en revanche, un lecteur s'appuyait sur la lecture des Relations antérieures pour conclure que cette visite des bourgades illinoises avait été décidée et convenue de longue date avec les Illinois eux-mêmes (ou un groupe d'entre eux), la mention d'une "robe noire» explique et justifie la décision prise par les deux hommes de prendre les devants et minimise du même coup la hardiesse alléguée de leur décision.

Deux lectures contradictoires sont ainsi permises dans ce passage, selon que l'on a en tête ou non les dernières Relations des jésuites publiées. Selon la première éventualité, les voyageurs savaient où ils allaient: ils étaient depuis longtemps attendus par des nations de langue algonquine (Illinois ou Miamis) vivant sur un affluent occidental du Mississippi, à «quelques

54. Recueil, p. 14-15. Mes italiques.

55. F. B. Steck relève également l'invraisemblance de ce passage dans Marquette Legends, p. 48.

56. F. B. Steck souligne aussi cet épisode qu'il considère curieux dans Marquette Legends, p. 51-52. 
jours » à peine de la mission Saint-François-Xavier; selon la seconde, les voyageurs auraient emprunté seuls un petit sentier, découvert par hasard et menant à un village dont ils avaient ignoré l'existence jusqu'alors.

\section{Un séjour bref ?}

Tout comme les longues descriptions de la «baie des Puants» et ses marées ${ }^{57}$ ou de la danse du calumet ${ }^{58}$ servent à étoffer un début de narration imprécis, les descriptions "curieuses " proposées au lecteur à l'occasion de ce premier arrêt des voyageurs font oublier le contexte et les objectifs réels de l'exploration.

À y regarder de plus près, l'épisode de ce séjour est effectivement lacunaire: alors que Jolliet et Marquette, si l'on en croit le récit de 1681 et les autres sources sur cette expédition, laissent canots et équipage sur une rive du Mississippi pour prendre le sentier menant au premier village, on retrouve tous les membres de l'expédition rassemblés lors de leur départ de ce village, après la cérémonie du calumet. Entretemps, aucune mention n'aura été faite du moment où les deux chefs retrouvent les cinq canoteurs censés être restés au bord du Mississippi. Ces derniers ont-ils rejoint Jolliet et Marquette en remontant la rivière pour transporter plus facilement les marchandises? Quand ont-ils eu l'assurance qu'ils pouvaient le faire sans danger? L'ont-ils fait avant l'invitation faite à Jolliet et Marquette de se transporter à la rencontre du "vrai chef » illinois? Jolliet et Marquette sont-ils plutôt retournés par le même sentier rejoindre leurs hommes après la cérémonie? Il est impossible de se faire une idée précise du déroulement de ce séjour à partir des renseignements fournis par la narration ${ }^{59}$. Ces imprécisions narratives font ainsi contraste avec la minutie des descriptions proposées.

Bien plus, certains détails descriptifs contredisent la narration suggérant, par exemple, que le narrateur n'en est pas à sa première participation à la «danse du calumet», puisqu'il sait que «l'Hyver la ceremonie se fait dans une Cabane, l’Esté c'est en raze campagne», ou que la cérémonie sert à marquer des occasions diverses (guerre, paix, alliances, etc.):

57. Voir une description similaire dans JR, vol. 56, 1671-1672, p. 136-140.

58. Voir la description de la «dance de la pipe à prendre tabac» dans JR, vol. 51, 1666-1667, p. 44-46. La comparaison des descriptions de cette danse dans le récit de 1681 et cette Relation montre que cette dernière est plus longue et plus précise que la description de 1681, quoique les deux descriptions suivent la même structure globale.

59. L'absence de dates et d'indications géographiques précises rend ce séjour d'autant plus obscur. Dans Marquette Legends, F. B. Steck imagine, avec peut-être une pointe d'ironie, l'étonnement de tous le lendemain, au moment de la rencontre entre Autochtones et engagés restés sur les rives du Mississippi (p. 51-52). 
La dance du Calumet, qui est fort celebre parmy ces peuples, ne se fait que pour des sujets considerables; quelquefois c'est pour affermir la paix, ou se réünir pour quelque grande guerre; c'est d'autres fois pour une réjouïssance publique, tantost on en fait honneur à une Nation qu'on invite d'y assister, tantost ils s'en servent à la reception de quelque personne considerable, comme s'ils vouloient luy donner le divertissement du Bal ou de la Comedie; l'Hyver la ceremonie se fait dans une Cabane, l'Esté c'est en raze campagne ${ }^{60}$.

Comment le narrateur peut-il savoir tout cela s'il n'est resté qu'une seule journée et n'a assisté qu'en une seule occasion à ce genre de cérémonie? Là encore, la lecture des Relations antérieures contredit l'inférence qu'il est tentant de faire à la lecture de cette longue description, à savoir que cette cérémonie devrait être tout à fait inconnue jusqu'alors. Si la cérémonie était peut-être nouvelle pour Marquette, elle ne l'était pas pour les lecteurs assidus des Relations ni pour d'autres missionnaires plus aguerris: Allouez avait assisté à au moins deux cérémonies du même type, dont la dernière avait eu lieu à l'occasion de la visite de Dablon dans les missions outaouaises. Si la lecture du seul récit de 1681 laisse croire que la cérémonie du calumet était une première pour le missionnaire qui la rapportait, comme cela devait être le cas pour le père Marquette, les détails de cette description et leur comparaison avec la Relation de 16701671 suggèrent plutôt que son narrateur avait participé à plusieurs de ces cérémonies à différentes saisons ou que, à tout le moins, il avait eu le temps de s'informer en profondeur sur le sujet (sur place ou en lisant les relations de ses confrères).

\section{Des événements dédoublés: double départ, double chemin de retour}

Encadrant la longue description des mœurs illinoises et de la cérémonie du calumet, la double mention du départ du village est elle-même curieuse : les voyageurs partent d'abord, avant cette description, «le lendemain» de leur rencontre avec le "grand Capitaine», ce qui n'alloue aux voyageurs qu'une seule nuit de repos; pourtant, la reprise de ce départ, plus loin dans la narration ${ }^{61}$, donne l'impression que deux séjours différents sont amalgamés ou qu’une portion du séjour n’a pas été rapportée,

60. Recueil, p. 24.

61. Encore une fois, F. B. Steck a remarqué cette incohérence et souligné l'heure de départ différente signalée pour chacune des occurrences. Il se peut que la traduction anglaise consultée par Steck ait été plus précise que la version publiée en français dans Thévenot, qui n’indique pas d'heure lors de la première mention du départ: c’est l'inférence que fait le lecteur qui établit ce départ dans la matinée, puisque le récit le mentionne juste après que les voyageurs eurent dormi dans «la cabane du Capitaine». 
le premier départ étant signalé avant que les voyageurs se déplacent à la rencontre du "grand capitaine» illinois :

Nous couchasmes dans la Cabane du Capitaine, $\&$ le lendemain nous prismes congé de luy, promettant de repasser par son Bourg dans quatre Lunes: Il nous conduisit jusques dans nos Canots, avec prés de six cens personnes qui nous virent embarquer, nous donnant toutes les marques qu' ils pouvoient de la joye que nôtre visite leur avoit causée. ${ }^{62}$

[...] Pendant que tous les Anciens petunoient apres nous pour nous honorer, on vint nous inviter de la part du grand Capitaine de tous les Ilinois, de nous transporter en sa Bourgade, où il vouloit tenir Conseil avec nous.

[Long développement sur le calumet et les mours illinoises]

Nous prenons congé de nos Ilinois sur la fin de Juin, vers les trois heures apres midy, nous nous embarquons à la veuë de tous ces peuples, qui admiroient nos petits Canots, n'en ayans jamais veu de semblables ${ }^{63}$.

Toutefois, le départ des voyageurs situé "vers la fin de juin" dans la deuxième mention, qui sert de transition entre la description et la reprise de la narration, est temporellement cohérent avec la dernière date mentionnée auparavant, soit le 17 juin $^{64}$, qui coïncidait avec l'arrivée des voyageurs à l'embouchure du Mississippi. Ce laps de temps alloue en effet aux voyageurs une dizaine de jours pour arriver chez les Illinois, ce qui correspond au temps nécessaire à des voyageurs en $\operatorname{canot}^{65}$. Toutefois, la datation approximative ne permet pas de savoir si les voyageurs étaient repartis aussitôt arrivés. Doit-on faire cas de la duplication de ce départ, qui peut passer pour une simple maladresse narrative? La réponse serait négative si elle n'était pas confirmée par d'autres incohérences du récit.

Ainsi, au moment de repartir, les voyageurs promettent aux Illinois péoria de revenir chez eux «dans quatre mois», ce que le récit confirme en conclusion par un aparté faisant mention d'un baptême réalisé au moment d'une seconde visite que le narrateur aurait faite sur le chemin du retour ${ }^{66}$.

62. Recueil, p. 19-20.

63. Recueil, p. 27-28.

64. Cette date est cependant variable selon les versions manuscrites de la Relation de 1673-1678.

65. Voir le calcul des milles parcourus et du temps nécessaire proposé par R. N. Hamilton dans Marquette's Explorations, p. 176-178.

66. Pour résoudre l'incongruité d'un passage similaire de la Relation qu'il examine, F. B. Steck se demande s'il est possible que les Péorias se soient installés à proximité des Kaskakias à la demande du missionnaire, dans Marquette Legends, p. 52-53, puis suppose que des Péorias se trouvaient alors chez les Kaskakias (Marquette Legends, p. 61), tout comme L. Kellogg l'a fait dans The French Regime, p. 197. 
Quand tout le Voyage n'auroit valu que le salut d'une ame, j'estimerois toutes mes peines bien recompensées, \& c'est ce que j’ay sujet de présumer; car lorsque je retournois nous passâmes par les Ilinois de Peroüacca, je fus trois jours à leur publier les mysteres de nostre Foy dans toutes leurs Cabannes, apres quoy comme nous nous embarquions, on m'apporta au bord de l'eau un enfant moribond que je baptisay un peu avant qu'il mourust, par une providence admirable, pour le salut de cette ame innocente ${ }^{67}$.

Cette seconde visite contredit pourtant la narration qui précède, car il est expliqué que les voyageurs ont emprunté, au retour, un chemin passant par la rivière Illinois qui leur «abrege de beaucoup le chemin ${ }^{68}$ ». La Carte de la Decouverte faite l'an 1673 dans l'Amerique Septentrionale jointe au Recueil de Thévenot contredit elle aussi cette promesse de retour et la conclusion du texte puisqu'elle indique un "chemin de l'allée», qui part du fond de la baie des Puants, emprunte la rivière Wisconsin, tandis qu'un "chemin du retour" prend naissance à la hauteur approximative de la rivière Des Moines et traverse les terres d'ouest en est pour rejoindre le cours supérieur de la rivière Illinois (Figure 1). De même, les dates de retour différentes indiquées par le Récit Des Voyages et des Decouvertes $\mathrm{Du}$ P. Jacques Marquette ${ }^{69}$ (identique en cela au Recueil de Thévenot) et la Relation de la découverte de la mer du Sud datée du $1^{\mathrm{er}}$ août $1674^{70}$, censée avoir été écrite à partir du témoignage de Jolliet ${ }^{71}$, ne coïncident pas : selon le premier texte, ce retour est effectif «sur la fin de Septembre ${ }^{72}$ »; selon le deuxième, il survient en novembre. Si Raphaël N. Hamilton se sert d’un passage du journal du dernier voyage de Marquette en 1674-1675 pour avancer que la date de novembre est erronée ${ }^{73}$, ce même passage est ici l'occasion de soulever une autre incongruité du récit de 1681 par rapport, cette fois-ci, au journal du second voyage du père Marquette. Quand, le 31 mars 1675 , ce dernier arrive au portage permettant la communication entre la rivière Des Plaines et la rivière Chicago où résident les Illinois kaskakia qu'il doit visiter, il se remémore son voyage précédent en évoquant le début du portage des voyageurs, comme si toute son aventure

67. Recueil, p. 43.

68. Recueil, p. 42.

69. AJC, Q-0001, 296, p. 37.

70. AFCJ, fonds Brotier 155, p. 12.

71. R. N. Hamilton, comme Ernest Gagnon avant lui, considère que la Relation de la mer du Sud du $1^{\mathrm{er}}$ août 1674 a été écrite sous la dictée de Jolliet, mais un examen plus attentif de ce texte montre que si le témoignage de Jolliet a certainement été pris en compte, il ne constitue pas l'unique source d'informations de Dablon, comme l'a montré Steck dans ses deux ouvrages.

72. R. N. Hamilton, Marquette's Explorations, p. 180-181.

73. R. N. Hamilton, Marquette's Explorations, p. 183. 


\section{Figure I}

\section{Carte de la decouverte faite l'an I673 dans l'Amerique Septentrionale}

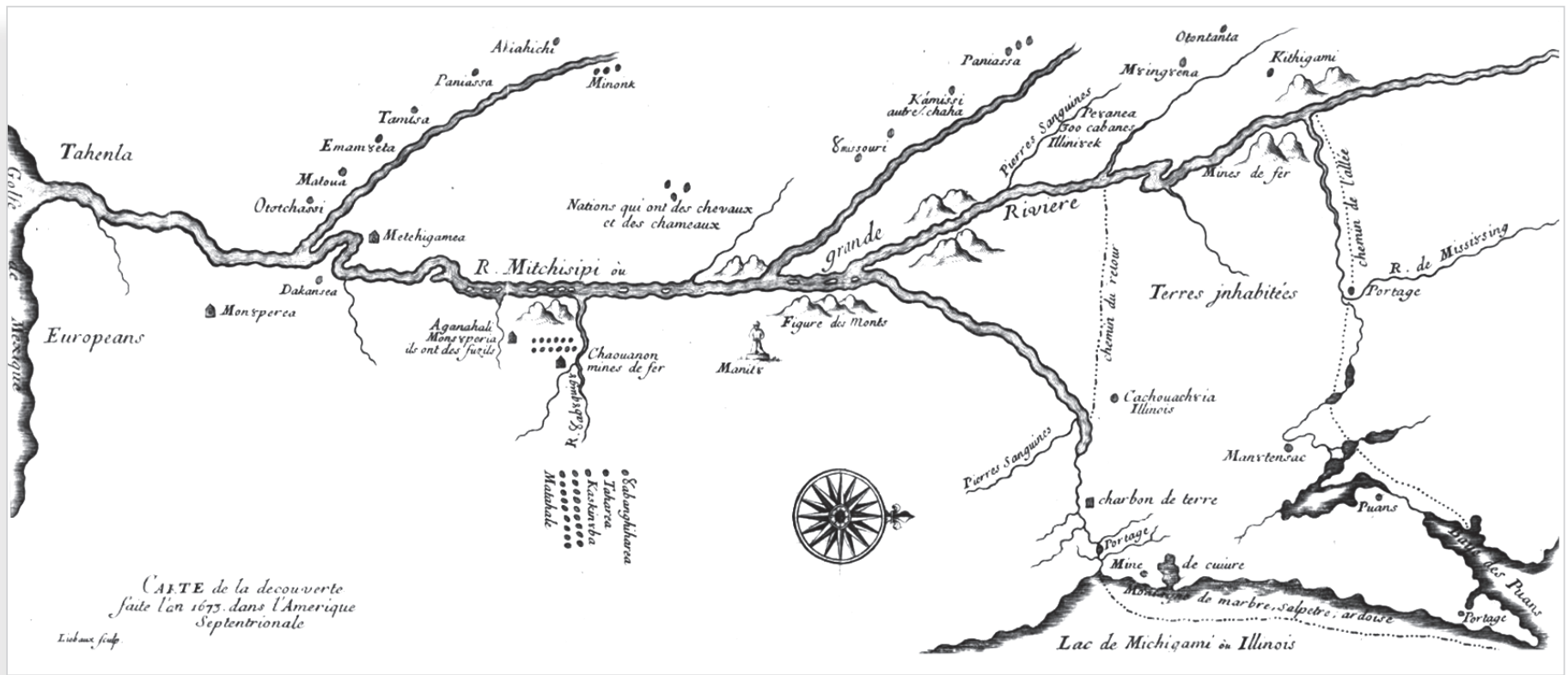

Source: Melchisedech Thévenot, Recueil de voyages, tome III. 
avait commencé là : "ce fust d'icy que nous commencasmes notre portage il y a $18 \mathrm{mois}^{74}$ ». Une telle formulation surprend, encore une fois, si le voyage de 1673 s'était bel et bien terminé par ce portage qui aurait permis aux voyageurs de boucler leur périple par le lac Michigan. Même le "chemin du retour» déjà signalé sur la carte proposée dans le Recueil, qui contredit et le texte de ce même Recueil et la Relation de 1673-1679, n'explique pas un début de portage si mémorable, à moins que ce chemin du retour corresponde en réalité à un "chemin de l’allée».

Compte tenu de ces deux itinéraires de retour concurrents établis par la carte et le récit de 1681, ce dernier synthétiserait non pas un voyage, mais deux, voire plus, ce que confortent et les titres inscrits sur deux cartes dites de la Manitounie ou Manitoumie $(1672,1673 \text { et } 1674)^{75}$ et la lettre de Marquette à Dablon de 1674, où le missionnaire signalait à son supérieur son retour à la mission de Saint-François-Xavier au printemps 1674 :

Ayant este contraint de demeuree a St. François tout l'este, a cause de quelque incommodité, en ayant este guery dez le mois de Septembre I’y attendois l'arriuee de nos gens au retour de la bas pour sçavoir ce que ie ferois pour mon hyvernement; lesquels m'en apporterent les ordres pour mon voyage a la mission de la Conception des Ilinois ${ }^{76}$.

Si tel était le cas, il faudrait alors envisager que deux voyages différents aient été faits ou qu'un second missionnaire (ou Jolliet seul) se soit chargé de l'exploration d'une partie du fleuve en aval du Missouri, avant de retourner au lac Michigan par la rivière des Illinois. Les dédoublements du départ de chez les Péorias et du retour vers la mission Sainte-Marie s'expliqueraient alors par la suppression de fragments de texte et l'amalgame de plusieurs narrations.

Cette hypothèse est confortée par une nouvelle incongruité : alors que la narration fait silence sur le narrateur même, le nom du jésuite Claude Allouez est convoqué deux fois par le texte, sans pourtant que soit mentionnée une quelconque participation directe de sa part à l'expédition de 1673.

74. AJC, Q-0001, 296, p. 68.

75. Carte de la nouvelle decouverte que les peres Iesuistes ont fait en l'année 1672; et continuée par le P. Iacques Marquette de la mesme compagnie, accompagné de quelq. Francois, en l'année 1673, qu'on pourra nommer en francois la Manitoumie [...], BNF, Estampes, 20, Vd 30; et Carte [...] Manitounie, BNF, Département Cartes et plans, CPL GE C-5014 (RES).

76. AJC, Q-0001, 296, p. 63. R. N. Hamilton, Marquette's Explorations, p. 121-124, se base sur la traduction anglaise de cet extrait pour nier que le retour de Marquette ait été aussi tardif en soulignant l'ambiguité du texte. Or si la traduction anglaise est ambiguë, le texte français ne l'est absolument pas. 


\section{En sourdine du texte: le jésuite Claude Allouez}

À l'instar de l'épisode de la cérémonie du calumet, d'autres descriptions s'insèrent dans des ellipses narratives et, ce faisant, les masquent. Avant d'arriver à l'agglomération des Miamis-Mascoutens-Kikapous (la mission Saint-Jacques), le narrateur remarque une plante médicinale que lui aurait signalée, à un moment non précisé, le missionnaire Claude Allouez. Cette mention d'Allouez, qui survient au moment où les voyageurs parviennent à la mission dont il est chargé, n'est guère étonnante. Plus étrange est le fait que ce missionnaire ait été absent lors du passage de son confrère, ce que le récit suggère pourtant en parlant à l'imparfait de son travail dans ce village, comme si Allouez avait alors cessé d'instruire les habitants de cette mission:

Ce Bourg est composé de toutes sortes de Nations qui s'y sont ramassées; des Miamis, des Maskoutens, $\&$ des Kikabeux: Les premiers sont les plus civils, les plus liberaux $\&$ les mieux faits; ils [...] ont parû si avides d'entendre le P. Alloües quand il les instruisoit, qu'ils luy donnoient peu de repos mesme pendant la nuit ${ }^{77}$.

La mention d'Allouez comme détenteur d'un savoir botanique essentiel surprend donc dans un texte qui, par ailleurs, laisse entendre que les voyageurs n'ont rencontré personne sur leur route depuis la mission de SaintIgnace, pas même Allouez, responsable de la mission par laquelle les voyageurs devaient passer ${ }^{78}$. Où est ce père au moment où les voyageurs passent dans sa mission? Celui-ci a-t-il montré au père Marquette la plante médicinale souveraine contre la morsure du serpent à sonnette lors du passage de ces derniers? A-t-il participé au conseil convoqué pour obtenir des guides Miamis? Comment envisager que, pour cette expédition préparée de longue date, Allouez n'ait pas été présent pour influer sur une négociation dont dépendait la réalisation du projet jésuite, alors même qu'il était présent dans cette mission à peine un mois auparavant ${ }^{79}$ ?

Si l'on en croit la Relation de 1672-1673, Allouez aurait passé trois mois (du 3 février au 30 avril 1673) chez les Outagamis avant de repartir pour les Mascoutens où il serait arrivé le 4 mai, et il serait à nouveau reparti de chez les Miamis d'où une extinction de voix l'aurait poussé à repartir pour une destination inconnue le $« 22^{\mathrm{e}} »$ :

77. Recueil, p. 6-7. Mes italiques.

78. F. B. Steck s'était également étonné du silence du récit de 1681 sur le travail apostolique des missionnaires, Marquette Legends, p. 44.

79. $J R$, vol. $58,1672-1673$, p. 62 . 
J'ay visité vne bonne partie des miami, ils ont de grandes Cabanes de grosses escorce, ou ie Les ay fait prier Dieu, tout Le monde a genoux iusques a ce que ie m'enrhumay soit La, soit dans nostre Chapelle toute entrouuerte si bien que ie ne pouuois presque plus parler, ie m'en allay Le $22^{280}$.

Un raisonnement par inférence, à partir de la dernière date complète mentionnée, soit le 4 mai 1673, laisserait croire que le missionnaire aurait quitté des Miamis habitant avec les Maskoutens au mois de mai (ce dont il est impossible d'être sûr, puisque la version originale de la lettre envoyée par Allouez à Dablon a pu être filtrée par Dablon et qu'un passage de cette lettre aurait pu être supprimé). Quoiqu’il en soit, cette même Relation ne donne aucune information sur les activités subséquentes du missionnaire. Si ce dernier était absent de la mission de Saint-Jacques des Maskoutens lors du passage des voyageurs, il aurait montré à son collègue le "simple » décrit dans le récit de 1681 lors d'un voyage ou d'un séjour précédent où les deux pères se seraient côtoyés, afin que Marquette soit en mesure de reconnaître la plante. Or Marquette se révèle effectivement en mesure de distinguer cette dernière des autres plantes poussant le long des berges, ce qui contredit le besoin qu'il aurait éprouvé, d’après le récit de 1681, de «l'examiner». Cet examen aurait dû, en toute logique, se faire au moment même de l'initiation du père Marquette aux valeurs médicinales de la plante en question, afin que, justement, il puisse la reconnaître par la suite. On pourra alléguer qu'un tel raisonnement fait abstraction de la curiosité très légitime qui pouvait animer Marquette, ou tout simplement que la véritable raison pour cueillir cette plante était autre : le père aurait voulu se prémunir contre une morsure mortelle, la plante étant censée constituer un remède contre la morsure des serpents à sonnette et un répulsif efficace contre ces reptiles. Ces objections n'expliquent cependant pas le jeu d'absence-présence qu'instaure le récit, ni ne permettent de savoir si Allouez était ou non dans sa mission au moment où sont passés les voyageurs.

En revanche, un passage du Voiage fait du Canada, rédigé en 1684-1685 par l'ingénieur Jean-Baptiste Minet à partir du témoignage de deux engagés de René-Robert Cavelier de La Salle sur leur expédition au Mississippi en 1682, nous met sur la piste d'une explication en affirmant, curieusement, que deux missionnaires auraient participé à l'expédition de Jolliet ${ }^{81}$.

80. JR, vol. 58, 1672-1673, p. 62 .

81. J.-B. Minet déclare s'être basé sur les témoignages de Gabriel Barbier et Nicolas de La Salle, qu'il aurait pris en note, pour rédiger son Voiage fait du Canada par dedans les terres allant vers le sud dans l'année 1682, BAC, MG18-B19. 
il [Cavelier de La Salle] vint en France, pour demander de faire un commerce de pelterie du costé de son fort, et d'aller faire un establisement au Ilinois; quoy qu'il ni eut pas encore esté ne sçachant que c'estoit un bon pays, que par le rapport des Canadiens qui y vont en traitte, et d'un nommé Mr. Joliet [ajout marginal: et deux jésuites] qui le premier en avoit fait la decouverte ${ }^{82}$.

Si tel était le cas, la première personne du pluriel utilisée par Marquette pour parler du voyage qu'il projette en 1670 pourrait prendre un nouveau sens :

Si les Sauvages qui me promettent de faire un Canot, ne me manquent point de parole, nous irons dans cette Riviere tant que nous pourrons, avec un François, $\&$ ce jeune homme qu'on m’a donné, qui sçait quelques-unes de ces langues, \& qui a une facilité pour apprendre les autres, nous visiterons les Nations qui les habitent, afin d'ouvrir le passage à tant de nos Peres, qui attendent ce bon-heur il y a si long-temps, cette découverte nous donnera une entiere connoissance de la Mer ou du Sud, ou de l'Oüest ${ }^{83}$.

Le nous que l'on pouvait prendre pour un pluriel englobant le groupe ou la mission pourrait ajouter au me du narrateur un compagnon déjà prévu, qu'il serait inutile de nommer puisqu'il est connu du supérieur jésuite. L'existence de deux pères pour l'expédition de 1673 correspondrait alors aux deux objectifs annoncés dans les Relations antérieures: l'un aurait pu vaquer à une mission illinoise à l'ouest du Mississippi, tandis que l'autre pouvait aller plus loin pour ouvrir de nouveaux champs apostoliques.

\section{La rivière Pekitanoui : un itinéraire étrange}

Enfin, une incongruité quant à l'itinéraire emprunté par les voyageurs entre le village des Péorias et le Missouri se révèle à l'examen des différentes versions du récit de cette expédition ${ }^{84}$. Dans celle de 1681, les voyageurs se retrouvent à descendre le Missouri après leur départ des Illinois:

Nous prenons congé de nos Ilinois sur la fin de Juin, vers les trois heures apres midy, nous nous embarquons à la veuë de tous ces peuples, qui admiroient nos petits Canots, n'en ayans jamais veu de semblables.

82. J.-B. Minet, Voiage fait du Canada, p. 3-4.

83. JR, vol. 54, 1669-1670, lettre de Marquette à Le Mercier, p. 188-190. Mes italiques.

84. R. N. Hamilton, qui relève également cette incongruité, l'attribue à une erreur de lecture de la part de Dablon, erreur qui serait due à une mauvaise ponctuation du manuscrit (p. 134). Néanmoins, si la modification qu'il préconise (l'ajout d'un point après le mot "courant») permet de résoudre l'ambiguiité de la traduction anglaise avec laquelle Hamilton travaille, elle ne résout pas celle que l'on relève dans le texte français, qui se situe davantage du côté des référents (de laquelle et cette rivière: de quelle rivière parle-t-on dans chacun des cas?). Sa correction créerait même une phrase syntaxiquement bancale. 
Nous descendons suivant le courant de la riviere appellée Pekitanoni ${ }^{85}$, qui se décharge dans Mississipy venant du Nord-Oüest, de laquelle j'ay quelque chose de considerable à dire, apres que j’auray raconté ce que j’ay remarqué sur cette riviere ${ }^{86}$.

Les voyageurs ont-ils vraiment descendu la rivière Missouri avant d'arriver au Mississippi, ce qui supposerait qu'ils l'aient atteint en remontant la rivière Des Moines puis en portageant pour rejoindre un affluent secondaire qui y aurait mené ${ }^{87}$ ? Le fait que, dans le récit de 1681 comme dans les autres versions de l'exploration de 1673 , la rivière Illinois soit signalée non pas à l'aller, lors de la descente du fleuve, mais au retour ${ }^{88}$, conforte le caractère vraisemblable d'une telle hypothèse.

\section{CONCLUSION}

En somme, deux objectifs distincts se profilent dans les Relations annuelles des jésuites jusqu'en 1672 pour l'expédition projetée au Mississippi et au-delà : l'ouverture d'une mission chez des Illinois vivant sur la rive occidentale du fleuve, le long d'un affluent correspondant vraisemblablement à la rivière Des Moines, et l'exploration d'un second affluent important menant très loin vers l'ouest, dont l'embouchure sur le Mississippi était située à environ 6 à 7 jours plus au sud (sans doute le Missouri). Pourtant, le récit publié en $1681^{89}$ passe sous silence ces objectifs, insiste sur la bravoure des voyageurs et laisse croire à un séjour très bref, donc de peu de conséquence, chez les Illinois péoria; de plus, la narration passe outre le Missouri, sans pour autant que les voyageurs prennent la peine de vérifier dans quelle

85. Ce toponyme ne se trouve ni dans la lettre ni dans la carte attribuée à Jolliet. La raison qu'en donne F. B. Steck dans The Jolliet-Marquette Expedition 1673, p. 20, est que Jolliet l'aurait oublié. Cette affirmation procède sans doute d'une inférence tirée d'un passage la Relation de la mer du Sud du $1^{\text {er }}$ août 1674 présentant ainsi le témoignage de Jolliet: «Voicy Toutes fois ce que nous en auons pû recueillir de ce qu'il nous a raconté» (transcription tirée de J. Delanglez, Louis Jolliet, p. 398). L’idée que Jolliet ait eu une piètre mémoire paraît difficile à endosser, ne serait-ce que parce que Jolliet avait fait des études et était musicien, activités qui sollicitent activement la mémoire.

86. Recueil, p. 27-28. Mes italiques. La même incongruité se retrouve dans la version du manuscrit de la Relation de 1673-1678 conservé à Montréal et dans les copies conservées aux AFCJ, fonds Brotier 149 ( $\left.f^{\circ} 22\right), 159$ ( $f^{\circ} 43$ ) et 158 ( $f^{\circ} 36 v$ ), à la différence près que le nom autochtone donné à la rivière Missouri y est écrit «Pekitanoui» et non «Pekitanoni».

87. La Carte d'Amérique dressée pour l'usage du Roy dessinée par Guillaume Delisle en 1722, par exemple, détaille le cours du Mississippi et indique une voie d'eau permettant un passage du bassin de la Des Moines à celui du Missouri moyennant un portage relativement court. Sa carte de 1718 indique aussi un "chemin des voyageurs» traversant d'est en ouest le territoire depuis le Mississippi jusqu'au Missouri.

88. F. B. Steck souligne également le fait que les voyageurs ne semblent pas remarquer l'embouchure de la rivière Illinois lors de la descente du fleuve, mais l'explique par le fait que les voyageurs devaient suivre la rive occidentale, Marquette Legends, p. 53.

89. Comme d'ailleurs les différentes copies de la Relation de 1673-1678. 
mer le Mississippi se décharge. Les voyageurs «descendent» néanmoins cet affluent important, ce que l'on a expliqué, quand l'incongruité a été soulignée, par l'intervention maladroite d'un copiste inattentif.

Dans ce récit où seul Jolliet est explicitement nommé, tandis que le nom de Jacques Marquette apparaissant dans la table des matières et l'Avis au lecteur incite ce dernier à associer Marquette au narrateur, Claude Allouez, protagoniste indirect mais néanmoins important (c'est le seul autre personnage nommé dans le Récit) demeure dans l'ombre, alors que plusieurs indices laissent penser que son rédacteur aurait pu chercher à minimiser le rôle d'Allouez dans une ou des expéditions qui auraient impliqué non pas un, mais au moins deux missionnaires.

Une telle lecture cherchant à éclairer les lacunes du récit attribué à Marquette permet d'esquisser un scénario de voyage bien plus complexe et plus élaboré où figurent plusieurs missionnaires impliqués dans une exploration s'étalant sur plus d'un an, de nombreux villages visités lors d'une incursion vers l'occident à partir de la Des Moines et l'exploration d'une partie du Missouri. Dès lors, le silence ayant pesé sur cette expédition et, plus globalement, sur la Relation de 1673-1678 pourrait s'expliquer par l'ordre royal de 1674 autorisant Frontenac à borner l'expansion territoriale jésuite ${ }^{90}$ : un récit circonstancié des initiatives jésuites aurait mis la Compagnie dans l'embarras si les jésuites avaient outrepassé leurs prérogatives et présumé de la permission royale en créant sans attendre de nouvelles missions au-delà du grand fleuve. La véritable raison expliquant l'arrêt de la publication des Relations annuelles découlerait alors de l'exploration elle-même, impossible à raconter.

L'opération consistant à rapporter un voyage déterminant sans paraître désobéir aux ordres royaux était bien délicate. Si, huit ans après l'expédition, un rédacteur chevronné s'était risqué tout de même à compter sur la courte mémoire du lecteur pour publier un récit lacunaire et elliptique, mais «curieux», qui puisse contrer les prétentions de Cavelier de La Salle et assurer au moins à la Compagnie de Jésus la préséance dans une "découverte» majeure, celle du Mississippi, on comprendrait alors pourquoi le Récit est si réticent à endosser certains faits et laisse le lecteur se fourvoyer lui-même grâce à sa capacité d'inférence. On comprendrait également les multiples précautions ayant entouré cette publication.

90. Voir le Mémoire de Frontenac du 13 novembre 1673, ANOM, Série C11A, vol. 4, f $\mathrm{f}^{\mathrm{D}} 25 \mathrm{v}$. et la lettre de Colbert à Frontenac du 17 mai 1674, ANOM, Série B, vol. 6, fo 26r. 
Bien entendu, le jeu d'inférence et de déduction instauré par l'explicite et l'implicite de ce récit ne permet pas d'affirmer une quelconque nouvelle vérité concernant le voyage d'exploration de 1673. Il montre simplement que d'autres lectures sont possibles. Pour espérer démêler l'embrouillamini créé par l'écriture même des sources, manifestement délibérément ambigües, et sortir des a-priori de lecture établis depuis plus de 300 ans par un texte habile, il faudra examiner avec le même soin toutes les autres sources sur cette expédition et les confronter les unes aux autres. La lecture présentée ici a, à tout le moins, l'avantage de rendre justice à la complexité de la construction narrative et descriptive du récit publié dans Thévenot. Surtout, elle suscite de nouvelles questions et ouvre de nouvelles pistes de réflexion sur cet épisode charnière de l'histoire de la Nouvelle-France. 